\title{
Gegen den Wind segeln
}

\author{
Harald Walach \\ University of Northampton, School of Social Sciences \& Samueli Institute for Information Biology, Northampton, UK
}

Nicht dass ich jetzt ein alter Seebär wäre, eher eine Landratte mit Hang zum Bergsteigen. Aber in meiner Jugend hatte ich ein Segelerlebnis, an das ich mich dieser Tage erinnere: Ein Schulfreund von mir war ein guter Segler, lieh sich hin und wieder ein Segelboot aus und lud einmal ein paar von uns zum Segeln auf dem Ammersee ein. Der Ammersee ist zum Segeln sehr zu empfehlen: Man sieht die Berge, sollte in der Mitte des Sees das Boot untergehen, kann man zur Not noch ans Ufer schwimmen, und wenn man ans richtige Ufer schwimmt, lockt der heilige Berg Andechs mit seinem weltberühmten Bier. Das also war die bayrische Version der Hochseetauglichkeitsprüfung für mich.

Ich habe schnell begriffen, dass man sich hinauslehnen muss, um gegen den Wind zu halten, und den Kopf einziehen muss, wenn man in den Wind dreht, weil man sonst die Rahe an den Schädel bekommt. An jenem Pfingstmontag war der Ammersee sehr ruhig. Also trudelten wir in die Seemitte, um etwas mehr Wind einzufangen. Trotzdem kamen wir über Stunden kaum über ein müdes Geplätscher hinaus, das wir uns mit der einen oder anderen Flasche Bier untermalten. Plötzlich leuchteten überall Lichter. Witzig irgendwie: die Sonne scheint, alles ist still, kein Wölkchen am Himmel, die Berge sitzen wie hingepurzelt in der Ferne, und ringsum strahlen plötzlich Lichter auf. Sturmleuchten, sagt mein Freund. Kein Sturm weit und breit. Das ist, lerne ich, weil Sturmleuchten normalerweise bereits 1-2 Stunden, bevor es ernst wird, aufleuchten. Wir freuen uns daher über die auffrischende Brise und gedenken, noch ein bisschen hin und her zu kreuzen, bevor wir uns wieder ans heimatliche Ufer begeben. Innerhalb von Minuten wird es ernst: eine riesige Wolkenfront kriecht über den Dießener Hügel und verdunkelt die Sonne. Es wird rasch kalt. Eine heftige Windbö schubst uns unsanft aus dem Bierdusel und reißt mir fast das Seil aus der Hand. Ehe wir es richtig begreifen, ist der Sturm da. Blöderweise hatten wir, da so wenig Wind war, vorher die Genua- fock gehisst, die sich nun aufbläht wie ein Ballon. Da wir das Boot nicht kennen, können wir sie nicht rechtzeitig einholen, und mit dem Sturm in den Segeln und nur sechs Händen schaffen wir es gerade mal, nicht zu kentern. Das richtig Dumme ist: Wir müssen genau in die Richtung zurück, aus der der Wind kommt. So viel weiß ich mittlerweile: Gegen den Wind kreuzen ist an sich ganz lustig, aber aufwendiger als mit dem Wind segeln. Dauernd ist man steil am Wind, muss sich bei starkem Wind enorm anstrengen, das Gegengewicht zu halten, und bei einem Wendemanöver blitzschnell ducken und auf die andere Seite springen, noch bevor der Sturm Zeit hat, das Segel voll zu packen und das Boot zu kentern. Ringsum sehen wir stolze Jachten und kleine Jollen, so wie wir eine haben, kentern. Bestimmt 20 Boote in unserer Umgebung liegen Kiel oben, die Insassen bemüht, sich obenaufzusetzen und auf Rettung zu warten. Wir erfahren später aus der Zeitung, dass für manche die Rettungsaktion bis in den anderen Morgen gedauert hat. Am Boden kullern zwei Bierflaschen im Wasser und erinnern mich an das gestrandete Schiff vor der «Schatzinsel».

Irgendwie ist es uns gelungen, immer in kleinen Sturmpausen, wenn der Sturm etwas abflaute, wieder die Richtung gegen den Wind so zu wechseln, dass wir in weitem Zickzack auf das rettende Ufer zusteuerten. Bei jeder Wende jubelten wir, denn wir hatten dem Sturm ein kleines Schnippchen geschlagen. Nach vielleicht fünf Kreuzungsmanövern gerieten wir plötzlich in den Windschatten des Ufers, dann der Bäume und waren gerettet. Wir mussten eine zerfetzte Genuafock berappen, aber das war zu verkraften und vermutlich billiger, als von der Wasserwacht mit einem gekenterten Boot ans Ufer geschleppt zu werden.

Ich habe damals hautnah erlebt und plötzlich verstanden, was es heißt, «gegen den Wind» zu kreuzen. Es ist alles andere als ein Sonntagsspaziergang. Es kann sogar sehr gefährlich werden, wenn der Wind stark ist, und es ist deutlich langsamer, als

\begin{tabular}{|c|c|}
\hline KARGER & (C) 2008 S. Karger GmbH, Freiburg \\
\hline $\begin{array}{l}\text { Fax }+497614520714 \\
\text { E-mail Information@Karger.de } \\
\text { www.karger.com }\end{array}$ & $\begin{array}{l}\text { Accessible online at: } \\
\text { www.karger.com/fok }\end{array}$ \\
\hline
\end{tabular}

Prof. Dr. Dr. Harald Walach

University College Northampton

School of Social Sciences \& Samueli Institute for Information Biology

European Office Boughton Green Rd, UK-Northampton NN2 7AL

Tel. +44 1604-892952, Fax -722067

E-mail harald.walach@northampton.ac.uk 
mit dem Wind zu segeln. Aber es macht riesigen Spaß, wenn man es schafft.

Gegenwind erfährt die Komplementärmedizin dieser Tage an vielen Orten. Große Studien sind in manchen Gebieten durchgeführt worden. Manche waren methodisch exzellent und ihre Ergebnisse sprechen für die Komplementärmedizin. So etwa die deutschen Akupunkturstudien, die die Diskussion über die Erstattungsfähigkeit der Akupunktur wenigstens teilweise beendet haben [1-5]. Andere waren zwar vielleicht methodisch gut, aber nicht schlüssig und haben mehr Fragen aufgeworfen als beantwortet; so etwa unsere Fernheilstudie [6] oder die Münchner Amalgam-Studie [7]. Wieder andere waren nur scheinbar gut, aber haben Fragen beantwortet, die keiner gestellt hat. Das könnte man etwa von manchen der großen Phytopharmakastudien sagen [8]. Trotzdem, oder vielleicht sogar deswegen, hören wir in letzter Zeit häufiger denn je, dass Komplementärmedizin nicht wirksam sei, es keine vernünftige Basis für Beweise und Entscheidungen gebe und man solche Fächer nicht an Universitäten unterrichten dürfe (vgl. [9]). Gerade hier in England scheint eine gezielte Kampagne anzulaufen. Einige namhafte Professoren und Journalisten haben an Entscheidungsträger im Gesundheitssystem geschrieben und sie aufgefordert, Homöopathie und andere Verfahren aus der Versorgung zu nehmen, da sie nicht auf Beweisen fußen [10-12]. Mehr oder weniger die gleichen Autoren haben in Times Higher Education, einer englischen Wochenzeitschrift für akademische Belange, dazu aufgefordert, Postgraduiertenkurse in Komplementärmedizin abzuschaffen oder kritisch unter die Lupe zu nehmen, da sie angeblich nicht auf wissenschaftlich fundierten Inhalten aufbauen. Prof. Jütte und ich haben beim British Journal of Clinical Pharmacology einen Letter eingereicht, in dem wir unter anderem versucht haben, in ein paar Zeilen auf die inhärente Paradoxie des momentan gültigen Wirksamkeitsbegriffs hinzuweisen [13]. Als Beleg für diese Aussage haben wir die paradoxen Befunde der großen GERAC-Studien zitiert, die in zwei von drei Fällen eine signifikante Überlegenheit des Akupunkturplacebos über die konventionelle Therapie belegt haben und im dritten Fall die Gleichwertigkeit von Akupunkturplacebo und konventioneller Behandlung nahelegen [14-16]. Das Grundargument, das ich ja schon oft vertreten habe, lautet, dass das herkömmliche pharmakologische Wirksamkeitskonzept nicht als allgemeiner Maßstab brauchbar ist. Denn es setzt voraus, dass die Referenzgruppe, gegen die getestet wird, nämlich Placebo, immer etwa gleich bzw. kontext- und situationsinvariant ist. Diese Voraussetzung wurde jedoch erstens nirgendwo belegt und wird zweitens, wenn sie denn untersucht wird, nicht erfüllt. Wie zwei aktuelle Metaanalysen zu Antidepressiva [17, 18] zeigen, ist dies nicht nur ein Problem der Komplementärmedizin, sondern auch der konventionellen Pharmakologie. Beide Metaanalysen zeigen: Die wahre Effektstärke der neuen Generation von Serotonin-Wiederaufnahmehemmern (SSRIs) zur Behandlung von Depression ist etwa $\mathrm{d}=0,3$, ist also eigentlich gering und erreicht vor allem nicht die pragmatische
Schwelle klinischer Bedeutsamkeit, die oft bei $\mathrm{d}=0,5$ angesetzt wird. (Diese Schwelle setzt das National Institute for Clinical Excellence (NICE) beispielsweise bei Antidepressiva.) Die eine Analyse zeigt: die oft gefundene Aufblähung von Effektstärken konventioneller Pharmaka kommt dadurch zustande, dass negative Ergebnisse häufig nicht veröffentlicht werden (Publikationsbias) (vgl. [19]). Die andere Analyse zeigt: die vermeintliche Überlegenheit von SSRIs über Placebo kommt einzig und allein dadurch zustande, dass die Placebowirkung bei schwer Depressiven zurückgeht, während die Pharmakawirkung immer ungefähr gleich groß ist. Die konventionelle Pharmakologie leidet also unter den gleichen $\mathrm{Pa}$ radoxien wie die Komplementärmedizin und hat ebenfalls mit der inhärenten Inkonsistenz des Wirksamkeitsbegriffs zu kämpfen. Unser Letter wurde selbstredend abgelehnt. Einer der Reviewer schrieb, was wir zum Thema Wirksamkeit zu sagen hätten, sei nicht akzeptabel. Die herkömmliche Pharmakologie habe einen sehr klaren, eindeutigen Wirksamkeitsbegriff, mit dem sie keine Probleme habe.

Ich finde es interessant, dass die Kampagne gegen die Komplementärmedizin zur gleichen Zeit beginnt, zu der sich zeigt, dass Flaggschiffmedikationen der konventionellen Pharmabranche in ihrer Glaubwürdigkeit bröckeln. Die Metaanalysen der SSRIs haben nicht nur gezeigt, dass ihre Effektstärke und klinische Nützlichkeit gering sind. Die größte aller OutcomesStudien, das STAR*D-Trial [20], hat darüber hinaus gezeigt, dass die Effekte mit einer großen Zahl von Nebenwirkungen eigentlich zu teuer und mit zu geringer Aussicht auf Langzeiterfolg erkauft werden [21]. Die Liste der erklärten Interessenskonflikte am Ende der STAR*D-Publikationen liest sich wie die weltweiten Gelben Seiten der Pharmabranche und ist in etwa genauso vollständig. NICE, die englische Behörde, die den Zugang von Arzneimitteln in die öffentliche Versorgung regelt, hat es vor Kurzem abgelehnt, die neue Generation von zentralnervös wirksamen Demenzmedikationen über öffentliche Gelder zu finanzieren (vgl. www.nice.org.uk/TA111). Die Wirksamkeit sei zwar belegt, aber die klinische Brauchbarkeit, also die Effizienz, zu gering (vgl. [22]). Der Direktor von NICE, Lord Michael Rawlins, hat in einem öffentlichen Vortrag des King's Fund zu Forschungsstrategien in der Komplementärmedizin im Oktober 2007 in London gesagt, dass es ihn nicht interessiere, welche Mechanismen wirksam sind, solange eine Intervention funktioniert und effektiv ist. Ein starkes Statement, das zeigt, dass das Umdenken weg von einem rein pharmakologischen Wirkmodell hin zu einem ganzheitlicheren Effizienzmodell nun auch in die Köpfe der Regulatoren gelangt ist.

Ist es möglich, fragt sich der neugierige, offene, und vielleicht etwas phantasiebegabte Zeitgenosse, dass da eine ganze Industriesparte unter Druck gerät, langfristig ihre Felle davonschwimmen sieht und beschließt, ein bisschen die Theaterwindmaschine anzuwerfen? Das dürfte nicht schwer sein. Ein paar emeritierte Pharmakologen zum Essen einladen vielleicht? Ein paar dümpelnde Arbeitsgruppen mit einem For- 
schungsauftrag bedienen? Jedenfalls Gegenwind erzeugen, wie auch immer. Ich würde mich nicht wundern. Die Koinzidenz ist bemerkenswert. Es ist nicht erstaunlich, dass jetzt Gegenwind entsteht. Die Komplementärmedizin ist endlich ein ernsthafter Gegner geworden, eine Kraft, die es Ernst zu nehmen gilt. Daher vielleicht der Wind. Gegen den gilt es zu segeln. Schopenhauer wird der Aphorismus zugeschrieben, dass jede Wahrheit durch drei Stufen geht: Erst wird sie ignoriert und belächelt. Dann wird sie bekämpft. Dann wird sie als evident bezeichnet, und man wundert sich, dass man das nicht schon vorher so gesehen hat. Und ganz in diesem Sinne hat Gandhi gesagt: «First they laugh at you. Then they fight you. Then you win.»

Jetzt also herrscht Gegenwind. Wir können stolz darauf sein. Es bedeutet, wir werden ernst genommen. Komplementärmedizin wird offenbar als möglicher Wirtschaftsfaktor gesehen. Jetzt heißt es, gegen den Wind segeln. Das kann Spaß machen, vor allem wenn wir uns klarmachen: Dies ist Phase zwei, die dritte kommt vielleicht schon bald.

\section{Literatur}

1 Brinkhaus B, Witt CM, Jena S, Linde K, Streng A, Wagenpfeil S, Irnich D, Walther HU, Melchart D Willich SN: Acupuncture in patients with chronic low back pain: a randomized controlled trial. Arch Intern Med 2006;166:450-457.

$>_{2}$ Linde K, Streng A, Jürgens S, Hoppe A, Brinkhaus B, Witt C, Wagenpfeil S, Pfaffenrath V, Hammers MG, Weidenhammer W, Willich SN, Melchart D: Acupuncture for patients with migraine: A randomized controlled trial. J Am Med Assoc 2005; 293:2118-2125.

-3 Melchart D, Streng A, Hoppe A, Brinkhaus B, Witt C, Wagenpfeil S, Pfaffenrath V, Hammes M, Hummelsberger J, Irnich D, Weidenhammer W, Willich $\mathrm{SN}$, Linde K: Acupuncture in patients with tensiontype headache: randomised controlled trial. Br Med J 2005:331:376-382.

4 Witt C, Brinkhaus B, Jena S, Linde K, Streng A, Wagenpfeil S, Hummelsberger J, Walther HU, Melchart D, Willich SN: Acupuncture in patients with osteoarthritis of the knee: a randomised trial. Lancet 2005;366:136-143.

5 Witt C, Jena S, Brinkhaus B, Liecker B, Wegscheider K, Willich SN: Acupuncture for patients with chronic neck pain. Pain 2006;125:98-106.

6 Walach H, Bösch H, Lewith G, Naumann J, Schwarzer B, Haraldsson E, Wiesendanger $\mathrm{H}$, Nordmann A, Tomasson H, Prescott P, Bucher HC: Efficacy of distant healing in patients with chronic fatigue syndrome: A randomised controlled partially blinded trial (EUHEALS) Psychother Psychosom 2008;77:158-166.
7 Melchart D, Vogt S, Köhler W, Streng A, Weidenhammer W, Kremers L, Hickel R, Felgenhauer N, Zilker T, Wühr E, Halbach S: Treatment of health complaints attributed to amalgam. J Dent Res 2008:87:349-353.

8 Group HDTS: Effect of hypericum perforatum (St. John's wort) in major depressive disorder. A randomized controlled trial. J Am Med Assoc 2002; 287:1807-1814.

9 Lewith G: Book Review. Forsch Komplementmed 2008; $15: 174$.

10 Colquhoun D: Science degrees without the science. Nature 2007;446:373-374.

11 Frank L, Chantler C, Dixon M, Colquhoun D: Should NICE evaluate complementary and alternative medicine? Br Med J 2007;334:506-507.

12 Goldacre B: Benefits and risks of homeopathy. Lancet 2007;370:1672-1673.

13 Walach H: Das Wirksamkeitsparadox in der Komplementärmedizin. Forsch Komplement Klass Naturheilkd 2001;8:193-195.

14 Haake M, Muller HH, Schade-Brittinger C, Basler HD, Schafer H, Maier C, Endres HG, Trampisch HJ, Molsberger A: German Acupuncture Trials (GERAC) for chronic low back pain: randomized, multicenter, blinded, parallel-group trial with 3 groups. Arch Intern Med 2007;167:1892-1898.

15 Diener HC, Kronfeld K, Boewing G, Lungenhausen M, Maier C, Molsberger A, Tegenthoff M, Trampisch H-J, Zenz M, Meinert R, Group ftGMS: Efficacy of acupuncture for the prophylaxis of migraine: A multicentre randomised controlled clinical trial. Lancet Neurol 2006;5:310-316.
6 Scharf H-P, Mansmann U, Streitberger K, Witte S, Krämer J, Maier C, Trampisch HJ, Victor N: Acupuncture and knee osteoarthritis. Ann Intern Med 2006;145:12-20.

17 Turner EH, Matthews AM, Linardatos E, Tell RA Rosenthal R: Selective publication of antidepressant trials and its influence on apparent efficacy. N Engl J Med 2008;358:252-260.

18 Kirsch I, Deacon BJ, Huedo-Medina TB, Scoboria A, Moore TJ, Johnson BT: Initial severity and antidepressant benefits: A meta-analysis of data submitted to the food and drug administration. PLoS Med 2008;5:e45.

19 Walach H: Publikationsbias, der erste oder letzte Nagel in den Sarg der Evidence-Based Medicine? Kommentar zu Turner EH, Matthews AM, Linardatos E, Tell RA, Rosenthal R: Selective publication of antidepressant trials and its influence on apparent efficacy. N Engl J Med 2008;358:252-260. Forsch Komplementmed 2008;15:110-112.

20 Rush JA, Trivedi MH, Wisniewski SR, Nierenberg AA, Stewart JW, Warden D, Niederehe G, Thase ME, Lavori PF, Lebowitz BD, McGrath PJ, Rosenbaum JF, Sackeim HA, Kupfer DJ, Luther J, Fava $\mathrm{M}$ : Acute and longer-term outcomes in depressed outpatients requiring one or several treatment steps: A STAR*D report. Am J Psychiatry 2006 163:1905-1917.

21 Fava GA, Tomba E, Grandi S: The road to recovery from depression - don't drive today with yesterday's map. Psychother Psychosom 2007;76:260-265.

22 Walach H: Journal Club. Forsch Komplementmed 2008;15:49-54. 